1 Universidade do Estado do Rio de Janeiro (Uerj), Instituto de Medicina Social - Rio de Janeiro (RJ), Brasil.

Orcid: https://orcid. org/0000-0001-90323919

marcelabelardo@yahoo.

com.ar

2 Universidade do Estado do Rio de Janeiro (Uerj), Instituto de Medicina

Social - Rio de Janeiro (RJ),

Brasil.

Orcid: https://orcid

org/0000-0003-3606-

5853

kenneth@uerj.br

\section{Tamizaje masivo: una revisión de la literatura sociológica}

\author{
Population screening: a narrative review of the sociological literature
}

Marcela Beatriz Belardo', Kenneth Rochel de Camargo Junior²

DOI: 10.1590/0103-1104201811818

RESUMEN Se provee una revisión de la literatura sociológica a partir de 79 obras referentes a cribados de cáncer y se excluyeron los cribados genéticos prenatal y neonatal. El tamizaje implica el monitoreo de poblaciones aparentemente sanas para rastrear una determinada condición. El rastreo invirtió la concepción tradicional de comprensión sobre las enfermedades al disminuir los umbrales de definición de enfermedad y rompió con la distinción médica tradicional entre enfermos y sanos. A partir de entonces, la práctica clínica comenzó a buscar enfermedades en personas asintomáticas, y así también modificó los comportamientos de los pacientes, que pasaron a ser responsables del cuidado de sí mismos.

PALABRAS-CLAVE Tamizaje masivo. Neoplasias. Revisión. Factores sociológicos.

ABSTRACT A review of the sociological literature is provided from 79 works referring to screening for cancer and prenatal and neonatal genetic screening were excluded. Screening involves monitoring apparently healthy populations to track a certain condition. Screening reversed the traditional conception of understanding about diseases by lowering disease definition thresholds and breaking the traditional medical distinction between the sick and the healthy. Clinical practice began to look for diseases in asymptomatic people and thus also modified the behavior of the patients, who became responsible for the care of themselves.

KEYWORDS Mass screening. Neoplasms. Review. Sociological factors. 


\section{Introducción}

En las dos últimas décadas, se ha instalado en la agenda de la salud internacional una creciente preocupación por la prevalencia de las Enfermedades no Transmisibles (ENT) en los países de bajos y medianos ingresos, según la clasificación que realiza el Banco Mundial. Dentro de esta clasificación médica se encuentra el cáncer y, según las estadísticas, su incidencia en América Latina y el Caribe es inferior a la de regiones más desarrolladas en el mundo, pero la diferencia radica en que la mortalidad es notablemente mayor ${ }^{\mathbf{1}}$. Esto puede explicarse, en parte, por un diagnóstico de la enfermedad en etapas más avanzadas de su desarrollo junto con un menor acceso a los servicios de diagnóstico, detección y tratamiento.

Una de las recomendaciones de la Organización Mundial de la Salud (OMS) para la prevención y control de las ENT, y en particular del cáncer, es que los países de medianos y bajos ingresos fortalezcan sus sistemas de salud para mejorar el diagnóstico precoz con la implementación de tamizajes masivos para ciertos tipos de cáncer, como el cervicouterino, el de mama, el de colon-recto y el bucal2 ${ }^{2}$. El tamizaje masivo implica la observación y monitoreo de poblaciones aparentemente sanas para rastrear precozmente una determinada condición a través de diferentes exámenes, como las mamografías, ecografías o diferentes test. Existen dos tipos de tamizaje: el oportunístico y el organizado. El primero se da cuando una persona busca los servicios de salud por otro motivo y el profesional de salud aprovecha la oportunidad para rastrear la enfermedad. Por el contrario, en el segundo caso el profesional de salud invita a la persona de un determinado grupo (género, edad o mayor riesgo) a ser objeto de seguimiento durante la implementación del programa, por lo tanto, existe un mayor control de las acciones. Concebimos a los tamizajes tanto una intervención médica como una intervención de la salud pública, por lo que conllevan desafíos legales, éticos y sociales.
Este tipo de programas se viene implementando en los países desarrollados desde hace por lo menos tres décadas, con resultados muy dispares. En los últimos años, se produjeron fuertes polémicas en torno a su eficacia ${ }^{3}$, los sobrediagnósticos que los tamizajes producen ${ }^{4}$ y el uso de las tecnologías 5 ,6. Uno de los debates más importantes fue el que desató el screening de cáncer de mama con el uso de la mamografía $^{7}$, en el que las diversas partes interesadas (desde grupos de pacientes hasta responsables de políticas y administradores de servicios de salud, médicos clínicos, científicos de laboratorio y el complejo médico industrial-financiero) se involucraron acaloradamente. En 2011, se constituyó la Red de Institutos Nacionales de Cáncer, que depende de la Unión de Naciones Sudamericanas (Rinc/Unasur), organismo estructurante y operativo que articula entre instituciones públicas, de ámbito nacional, desarrollando o ejecutando programas para el control del cáncer en la región, entre los que se encuentra el cribado para el cáncer cervicouterino. De los países asociados, Argentina y Uruguay ya están implementando pruebas piloto de cribado masivo para el cáncer colorrectal y cervicouterino.

Asimismo, existe una prolífica literatura en el campo de la salud pública que discute diferentes aspectos de los tamizajes masivos. Solo por citar algunos ejemplos, se están desarrollando estudios que refieren a los sistemas de información y registro de cáncer de América Latina y el Caribe ${ }^{\mathbf{1 , 8}}$, el análisis de costo-efectividad de los programas de tamizaje nacionales ${ }^{9} \mathrm{o}$ se proponen mejoras en el diseño de esos programas ${ }^{10,11}$. La proliferación de estos estudios, a la par de la alianza cooperativa de los institutos nacionales de cáncer de los países de Sudamérica y los países asociados del Caribe, está indicando que este tipo de intervenciones preventivas se está imponiendo en las agendas sanitarias de la región, aunque son relativamente escasos los estudios que abordan los aspectos sociales y sus consecuencias.

Esta revisión busca exponer los aspectos sociales de los tamizajes masivos, que 
usualmente no se toman en consideración por la mayoría de la literatura, pero que tienen importantes consecuencias debido a que rompen con la lógica tradicional de comprensión de las enfermedades y modifican la práctica clínica y el cuidado de la salud por parte de los pacientes.

Con la intención de traer este debate al campo de la salud colectiva, se presenta un conjunto de asuntos descritos en la literatura anglosajona, que ha sido la que más ha publicado sobre los aspectos sociológicos de los tamizajes. El artículo no tiene el objetivo de ser una revisión exhaustiva de la totalidad de los trabajos sociológicos, sino de proveer una visión panorámica de los conceptos más significativos relativos a los aspectos mencionados. Se han incluido los trabajos sobre screenings poblacionales referentes al cáncer y se han excluido aquellos referidos a los cribados genéticos prenatal y neonatal.

El texto está dividido en dos partes. La primera refiere a una revisión de la literatura sociológica que trata las transformaciones que los tamizajes masivos produjeron en el conocimiento médico sobre enfermedades, en la práctica clínica y en la gestión de la salud y la enfermedad por parte de los pacientes. La segunda parte discute dichas transformaciones porque tiene la intención de aportar al debate actual, teniendo en consideración la decisión de las autoridades sanitarias de varios países de la región de iniciar la implementación de los tamizajes poblaciones para diferentes tipos de cáncer.

\section{Metodología}

El artículo provee una revisión de la literatura sociológica sobre tamizajes para el cáncer dando cuenta de tres aspectos: 1) la manera en que los tamizajes han modificado la compresión y construcción de enfermedades (conocimiento médico); 2) la forma en que han transformado la práctica clínica; y 3) los modos en que han cambiado las percepciones y cuidados de la salud por parte de los pacientes (gestión de la salud y la enfermedad). En este sentido, la revisión es una revisión crítica que resume la literatura sobre un tema y extrae conclusiones. Este tipo de revisión es útil porque reúne un volumen de literatura sobre un tema específico, lo condensa y sintetiza. Su propósito principal es proporcionar al lector los antecedentes de un tema para comprender el estado actual del conocimiento alcanzado y ayudar a destacar nuevos significados para futuras investigaciones. Puede inspirar ideas de investigación identificando brechas o inconsistencias en un cuerpo de conocimiento, ayudando así al investigador a determinar o definir preguntas o hipótesis de investigación y, también, puede servir al desarrollo de marcos conceptuales o teóricos ${ }^{12}$. Además, se diferencia de la revisión sistemática en que esta última utiliza un enfoque más riguroso y bien definido para revisar la literatura en un área temática específica, generalmente, para responder preguntas centradas en la práctica clínica. Las revisiones sistemáticas detallan el tiempo dentro del cual se seleccionó la literatura, así como los métodos utilizados para evaluar y sintetizar los hallazgos de los estudios en cuestión. Este tipo de revisiones utilizan criterios explícitos y rigurosos para identificar, evaluar críticamente y sintetizar toda la literatura sobre un tema en particular ${ }^{13}$. A diferencia de esta revisión, el propósito de una revisión sistemática es proporcionar una lista lo más completa posible de todos los estudios publicados e inéditos relacionados con un área temática en particular ${ }^{\mathbf{1 2}}$.

La búsqueda, la lectura y la selección de la literatura para esta revisión se realizaron entre los meses de marzo y agosto de 2017. La primera etapa consistió en la búsqueda de artículos en bases de datos en salud, como PubMed y Biblioteca Virtual en Salud (BVS), utilizándose los descriptores 'screening', 'tamizaje' y'cribado' para el cáncer de la última década. La búsqueda arrojó una cantidad descomunal de artículos, lo que entonces solo permitió explorar e identificar, grosso modo, los tipos de trabajos que abordan la 
temática: ensayos clínicos, estudios epidemiológicos y sobre implementación, costoefectividad y evaluación de los programas de screening. Luego se realizó una búsqueda en la base bibliográfica SciELO (Scientific Electronic Library Online), dado que es la base de datos que agrupa los artículos de origen iberoamericano, utilizándose los descriptores 'tamizaje', 'cribado' y 'rastreamento' (en portugués). El propósito de esta segunda búsqueda consistió en explorar la literatura procedente de América Latina y el Caribe, debido al interés que este tema está suscitando en los países de la región. El filtro utilizado en este caso fue por revistas, entre las que seleccionamos aquellas que pertenecen a las ciencias sociales y humanas en salud, como revistas de salud colectiva y salud pública (se excluyeron las especializadas en el campo biomédico). La búsqueda arrojó 49 artículos, todos dedicados a la implementación y evaluación de programas de screenings, temas que no son foco de atención para esta revisión, por lo tanto, fueron excluidos.

En la segunda etapa, se buscó en revistas de sociología y filosofía de la salud de origen anglosajón, debido a la mayor experiencia de esos países en cribados masivos. De estas, se seleccionaron cuatro consideradas pertinentes por el enfoque y los temas que frecuentemente discuten ('Sociology of Health \& Illness'; 'Critical and Public Health'; 'Medicine, Health Care and Philosophy'; 'Health, Risk \& Society'). Seguidamente, se utilizó el descriptor 'cancer screening' y el operador booleano NOT para excluir 'genetic' y 'pregnancy'. Esa búsqueda arrojó numerosos artículos que fueron revisados por título y resumen, en función de las tres dimensiones descritas. Una vez hecho lo anterior, se aplicó el método de revisión de Cohen ${ }^{\mathbf{1 4}}$ para dar cierto tipo de estructura durante el proceso de lectura y selección. Esta técnica simple consiste en realizar una 'vista previa', reconocer a través de preguntas las dimensiones establecidas, leer, resumir e indexar. A continuación, del universo sobre tamizajes se seleccionaron 13 artículos para su lectura completa: 'Sociology of Health \& Illness' (256/6); 'Critical and Public Health' (84/4); 'Medicine, Health Care and Philosophy' (69/0) y 'Health, Risk \& Society' (101/3). Se realizó un resumen inicial con las ideas principales de cada uno en función de las tres dimensiones de la revisión y, posteriormente, se realizó una revisión más sistemática y crítica del contenido, con la utilización del sistema de indexación por título del trabajo, autor y fecha. A partir de la lectura de esos artículos, se amplió la bibliografía a través de la 'técnica de bola de nieve' bibliográfica ${ }^{15}$. Por ejemplo, 'Sociology of Health \& Illness' publicó una serie monográfica titulada 'The Sociology of Medical Screening: Critical Perspectives, New Directions', de 2012, editada por Natalie Armstrong y Helen Eborall. La colección contiene 10 capítulos de distintos autores. De esos capítulos, se seleccionaron 4 por su pertinencia para esta revisión, incluyendo la introducción, escrita por Natalie Armstrong y Helen Eborall, que sirvió como puntapié inicial para continuar ampliando las referencias bibliográficas, en su mayoría capítulos de libros y libros. Después, se revisaron, seleccionaron y resumieron aquellos considerados pertinentes a la temática de este trabajo. La etapa final consistió en la inclusión de ideas clave y comentarios de cada artículo. Finalmente, 79 obras fueron las elegidas y utilizadas para componer esta revisión.

\section{Resultados}

\section{Monitoreo de poblaciones sanas du- rante el siglo XX}

Desde la década de 1990, se ha desarrollado una importante producción sociológica con el fin de comprender la 'nueva salud pública' y su paradigma dominante: el de la promoción de la salud ${ }^{16}$, que busca fomentar nuevas 
actitudes frente a la salud, de manera generalizada, dentro de la sociedad, diferenciándose de su antecesora, la salud preventiva, basada en un enfoque más individualista y liberal17. Si bien describir las diferencias y similitudes entre ambos paradigmas no forma parte de esta revisión, una categoría clave e ineludible es la que refiere al riesgo. Aunque el vocablo sigue siendo bastante ambiguo, conforme a ciertos abordajes sociológicos, la evaluación del riesgo que tiene una persona de enfermar vino a dominar todo el escenario técnico de elaboración de normas y medidas de prevención y de promoción de la salud ${ }^{\mathbf{1 7}}$. La noción de riesgo se utilizó para justificar ciertas medidas impulsadas para promocionar una vida más saludable al mismo tiempo que justificó la implementación de tamizajes en masa para prevenir ciertos tipos de enfermedades (cáncer de mama, próstata, cervicouterino, colorrectal) o determinadas condiciones rastreables por los tamizajes prenatal y neonatal.

Así que entender el origen de cualquier área del conocimiento, de una tecnología médica, de un paradigma o, como en este caso, de una estrategia de la salud pública, es importante para comprender mejor sus trayectorias, desdoblamientos y perspectivas. Según la literatura revisada, los primeros tamizajes masivos pueden rastrearse a principios del siglo XX, periodo en el que Raffle y Gray ${ }^{18}$ comparan los orígenes de los tamizajes en los Estados Unidos y en el Reino Unido, donde los médicos comienzan a promover la novedosa idea de las consultas periódicas de los pacientes sanos para encontrar signos de alguna futura enfermedad. Previo a las guerras mundiales, los tamizajes implementados estaban enfocados en dos grupos de la población: los escolares, en quienes se buscaban anomalías que podrían afectar la salud en la edad madura; y los jóvenes varones que ingresaban al servicio militar, en quienes se buscaban enfermedades como la tuberculosis, a través de los rayos X; la sífilis, a través del Test de Wasserman (uno de los primeros cribados disponible a principios del siglo $\mathrm{XX}$ ); o enfermedades mentales, a través de cuestionarios que se aplicaban a los soldados que habían participado en la Primera Guerra Mundial ${ }^{19}$. Con el fin de la Segunda Guerra Mundial, se produjo una rápida expansión de los tamizajes a la población civil. Y a partir de la década de 1940, se implementaron los exámenes de glucosa y orina, para detectar diabetes; el Papanicolaou, para la detección del cáncer cervicouterino, convirtiéndose en el modelo para el resto de los screenings ${ }^{20-22}$; y los exámenes mamográficos, para detectar cáncer 23-25. Por otro lado, la estrategia de observar y monitorear a poblaciones aparentemente sanas para rastrear una enfermedad o condición se constituye en la forma de intervención de la salud pública del siglo XX, aunque su modalidad fue transformándose a lo largo del tiempo, como examinamos a continuación.

\section{Tamizaje y conocimiento médico}

La observación y monitoreo de la población ha sido analizada desde diferentes marcos teóricos a través de conceptos como vigilan$\mathrm{cia}^{26,27}$, riesgo ${ }^{28}$, gobernanza ${ }^{29}$, (bio)medicalización ${ }^{30-32}$ y la medicina como institución de control social ${ }^{33}$. Esta vigilancia de la población por la medicina, particularmente a través de los cribados, fue rigurosamente analizada por David Armstrong, que, influenciado por las ideas de Michel Foucault -pero analizando el mundo anglosajón del siglo XX-, reflexiona sobre un aspecto que constituye el trasfondo de los tamizajes masivos: el conocimiento médico, al argumentar que la medicina no solo describe su objeto, el cuerpo, sino que lo construye como una realidad biológica invariable $\mathrm{y}$, a través de diversos acontecimientos médicos, muestra cómo la medicina ha fabricado un cuerpo esencialmente subjetivo y relativo, a diferencia del cuerpo discreto y pasivo del siglo $\mathrm{XIX}^{\mathbf{3 4}}$. Desde esa perspectiva, el autor analiza en sus diversas obras los supuestos 
que subyacen a la salud pública en los últimos dos siglos, desde las estrictas regulaciones de los cordones sanitarios hasta los espacios constantemente monitoreados por la nueva salud pública ${ }^{35}$, y da cuenta de la emergencia de una nueva medicina basada en la vigilancia de las poblaciones asintomáticas a principios del siglo XX, denominándola 'medicina de vigilancia'27, que implicó una reasignación de los espacios de la enfermedad al abordar cuestiones como la normalidad, el rediseño de la relación entre síntoma, signo y enfermedad, y la localización de la enfermedad fuera del espacio físico corporal.

En consecuencia, la aplicación de tamizajes a escala poblacional alteró tres aspectos significativos para la medicina en un contexto en el que crecían en importancia las enfermedades crónicas mientras se reducían las enfermedades infecto-contagiosas, a saber: la definición de enfermedad, o sea, qué es y qué no es enfermedad; la incorporación del concepto de 'probabilidad', es decir, la probabilidad que tiene una persona de enfermar; y el nuevo papel que ocupa la medicina en la sociedad.

Para Armstrong19, la medicina se basaba en un modelo lineal temporal de la enfermedad en el que era posible incidir en cualquier punto de su progresión. Precisamente, el objetivo principal de un tamizaje consistía en el escrutinio de los cuerpos de los ciudadanos para detectar precozmente numerosas enfermedades y, así, alterar su curso a través de las tecnologías disponibles. Sin embargo, este modelo se modificó a partir del estudio de Framinghan de 1948, puesto que demostró que un conjunto de factores de riesgo podían ser predictores de enfermedades cardiovasculares, lo que significó una ruptura con la manera tradicional de comprender la etiología de las enfermedades. Por consiguiente, al modificarse la comprensión de la etiología de algunas enfermedades, los screenings se adaptaron a la nueva definición de enfermedad y reforzaron dicha transformación. A partir de entonces, según Armstrong, los tamizajes comienzan a detectar riesgos más que identificar signos tempranos de una enfermedad, como ocurre en la prevención secundaria $^{36}$. La identificación de factores de riesgo (prevención primaria) dio lugar a una nueva era en la medicina y en la salud pública, ya que el concepto de enfermedad (nos referimos a enfermedades no transmisibles) se modificó al disminuir los umbrales para designarla. En este nuevo escenario, los factores de riesgo se tornaron equivalentes a enfermedad. A su vez, esta equivalencia impactó en el significado de la cura y la prevención, convirtiendo ambos términos en categorías borrosas cuyas fronteras se vuelven más imprecisas y menos definidas, como analizaron Starfield et al $\mathbf{l}^{\mathbf{3}}$.

Otro aspecto que los tamizajes evidenciaron es la incorporación de la idea de 'probabilidad' de enfermar. Este cambio se empezó a producir a partir del estudio de Doll y Bradford Hill ${ }^{37}$, que dio cuenta de la relación entre mortalidad y el hábito de fumar. A partir de esa asociación, se empezaron a percibir como entidades inseparables el hábito de fumar y el cáncer de pulmón. Los trabajos de Jackson ${ }^{38}$ y Lupton ${ }^{39}$ han analizado precisamente la percepción que la sociedad tiene no solo del fumador activo, sino también del pasivo. No obstante, como señala Armstrong ${ }^{19}$, cuando nos referimos a un factor de riesgo (fumar, abuso de alcohol, alto consumo de sodio, baja actividad física) no está ligado con la futura enfermedad, ni necesariamente existe una relación directa con ella, simplemente abre un espacio de posibilidad.

El último aspecto que los tamizajes han ayudado a modificar es el que tiene que ver con el rol de la medicina en la sociedad. A lo largo del siglo XX, el objeto de preocupación y ocupación de la medicina se fue desplazando de la población enferma -como fue su práctica tradicionalmente a casi la totalidad de la población. Esta nueva manera de interpretar y comprender las enfermedades es reforzada por los tamizajes masivos, que rompen con la distinción médica tradicional entre enfermos y sanos ${ }^{19}$. Por consiguiente, la lógica dicotómica por la que operaba la práctica clínica se desvanece. 


\section{Tamizaje y práctica clínica}

En la década de 1990, la sociología de la salud ha examinado el estado de salud de las poblaciones y los medios utilizados para mantener una buena salud en las sociedades occidentales ${ }^{\mathbf{1 6}, 39,40}$. Justamente uno de los aspectos que ha estudiado con rigurosidad es la consulta clínica periódica, modalidad en la que se materializan los tamizajes, ya sea aquel que se produce espontáneamente o cuando el profesional invita a su paciente a formar parte activa de un programa de tamizaje organizado. En cualquiera de las dos modalidades el principio central es que las personas deben poner sus cuerpos a disposición de los profesionales de la salud para una inspección regular aunque no presenten síntomas.

El primer aspecto a tener en cuenta es que, para que un tamizaje alcance los objetivos propuestos, la consulta clínica debe rutinizarse ${ }^{41}$. En este cambio de hábito radica la primera diferencia entre este modelo de atención y los tradicionales modelos diagnósticos en los que el paciente iba a la consulta clínica a exponer sus síntomas y, a partir de allí, se detectaba (o no) una enfermedad. No obstante, los tamizajes rompen con esa lógica porque es el médico el que busca a su población objetivo para implementar exámenes diagnósticos sobre una población asintomática (sana). En este sentido, la búsqueda de determinados tipos de enfermedades o condiciones a través de los exámenes produce una clasificación de las personas en dos grandes grupos: aquellas con probabilidad de desarrollar una determinada condición y aquellas en las que es improbable ${ }^{19}$. Y esta clasificación está basada en el concepto de riesgo, es decir, el riesgo que tienen determinados individuos de enfermar y aquellos que no lo tienen. Por lo tanto, la relación entre riesgo y las estrategias poblacionales diagnósticas es sinérgica: las personas son exhortadas a asistir a un examen porque se considera que están en riesgo de desarrollar una enfermedad o condición, y las estadísticas que se producen a través de sus resultados sirven para apoyar o reformular evaluaciones de patrones de riesgo en la población ${ }^{39}$. Esta nueva modalidad que se impone en la práctica clínica se fue desarrollando intensamente a partir de nuevas y más sofisticas tecnologías diagnósticas $\mathbf{4 2 , 4 3}$.

El segundo aspecto a considerar son las nuevas tecnologías para producir diagnósticos, puesto que cambian la percepción y la construcción de los mismos, es decir, una observación más profunda y detallada del cuerpo modifica las maneras de comprender lo normal y lo patológico y permite construir nuevos diagnósticos ${ }^{\mathbf{4 4 , 4 5}}$. A este respecto, existe un conjunto de trabajos que discute sobre los límites cada vez más difusos entre enfermedad y salud ${ }^{46-50}$. Son muchos los ejemplos de anormalidades, disfunciones o enfermedades que se diagnostican por tecnologías más y más sofisticadas, que hacen transparentes nuestros cuerpos. Además, continuamente se diagnostican enfermedades a personas que tienen niveles muy leves de disfunción, y que son completamente compatibles con una vida plena ${ }^{51}$. El resultado de esta agudeza diagnóstica es una definición ampliada de enfermedad, lo que hace que aumente el número de personas 'enfermas' y los problemas de sobrediagnóstico ${ }^{52}$. Aquí emerge el tercer aspecto que se modifica con los tamizajes: un resultado positivo no implica un diagnóstico, sino que significa que la persona tiene una mayor probabilidad de presentar determinada enfermedad. Este resultado desencadena una serie de exámenes hasta lograr un diagnóstico definitivo y frecuentemente genera un serio problema: el alto porcentaje de diagnósticos equivocados. Algunos pacientes son considerados libres de una enfermedad, pero, de hecho, la tienen (falsos negativos), y otros reciben un diagnóstico de enfermedad cuando, en verdad, no la tienen (falsos positivos). Esto sucede cuando el examen no posee una óptima sensibilidad y especificidad -propiedades inherentes a cada una de las pruebas de diagnóstico- para que dé como resultado pequeñas tasas de falso positivo y para que garantice que 
la persona realmente no tiene la enfermedad cuando el resultado es negativo. Ello se debe a que los test diagnósticos tienen una sensibilidad (capacidad para detectar a individuos con la enfermedad) y una especificidad (capacidad de excluir el diagnóstico en los casos de los no enfermos) que nunca son del $100 \%{ }^{53}$. Cuando se produce un falso positivo se realizan múltiples exámenes hasta que el diagnóstico original es descartado. La problemática del sobrediagnóstico y, por ende, del sobretratamiento (que, a diferencia del caso anterior, ocurre cuando el individuo es diagnosticado con una condición que no le causará síntomas, tampoco la muerte) es un debate polémico y actual en la literatura sobre los tamizajes masivos ${ }^{4,51,54}$.

Ante esta situación, los tamizajes masivos se implementan para detectar algunas enfermedades o condiciones muy puntuales. Actualmente, la OMS recomienda los tamizajes para rastrear el cáncer cervicouterino, de mama, colorrectal y bucal (y la inmunización para HPV). No está indicado un rastreo masivo para estómago, piel, próstata, pulmón, cavidad oral ni esófago, pero hasta hace poco tiempo, también se recomendaba el screening para el cáncer de próstata, con el test PSA, sin embargo la Preventive Service Task Force de Estados Unidos, en su revisión de 2012 de las recomendaciones de 2008, desaconsejó el screening mediante este tipo de prueba, considerando que no reducía la mortalidad por cáncer de próstata y un tratamiento innecesario podía causar más daño ${ }^{55}$. En Argentina, está bastante difundido este tipo de estudio en la población masculina, a pesar de que las últimas recomendaciones lo desalientan. No obstante, sigue siendo común que el médico y su paciente soliciten el test PSA, por lo que termina por convertirse en una especie de tamizaje por la puerta de atrás $\mathbf{5 6}$.

Las polémicas que generan los tamizajes son otro aspecto a tener en cuenta, dado que los debates dentro del mundo médico no solo involucran argumentos científicos y clínicos, sino también argumentos sociales ${ }^{57}$. En este contexto, el estudio de Gøtzsche y Nielsen ${ }^{7}$ causó una fuerte polémica durante años entre los investigadores, que realizaron una revisión sistemática del cribado con mamografías y concluyeron que, en un período de 10 años, 1 de cada 2000 mujeres que fueron invitadas a ser examinadas prolongará su vida, 10 se someterán a tratamientos innecesarios y correrán nuevos riesgos, y 200 tendrán que hacer frente al efecto psicológico de un falso positivo. Como señalaba Geoffrey Rose ${ }^{58}$, un gran número de personas sometidas a un pequeño riesgo puede generar más casos de enfermedad que un pequeño grupo de personas que tengan un alto riesgo.

\section{Tamizaje y gestión de la salud y enfermedad}

Si en la sección anterior describimos las transformaciones que se sucedieron en la práctica clínica, aquí nuestro foco de atención está puesto en las transformaciones que los tamizajes masivos produjeron en los pacientes, una vez que esa práctica se hizo hegemónica en la sociedad. Desde diferentes postulados teóricos y objetos específicos de investigación, se han examinado las consecuencias de la nueva salud pública (en la que ocupa un lugar importante el cribado poblacional). Para la práctica clínica, los screenings y diagnósticos precoces son entendidos como 'tecnologías presintomáticas'. Sin embargo, al considerar que un individuo está en riesgo, se requiere una constante vigilancia de su entorno, tanto interno como externo, así como de su comportamiento. Estar en riesgo ha configurado un dispositivo perfecto para poner a la población en un estado constante de preparación y conciencia en cuanto a su salud ${ }^{59}$.

Desde la teoría sociocultural, Lupton y Petersen 40 examinaron diferentes aspectos de la nueva salud pública; el que nos interesa para esta revisión está relacionado con los derechos de los ciudadanos y sus responsabilidades y obligaciones implícitas. A continuación, los autores parten de una definición de tipo ideal del ciudadano 'sano': 
aquellos que tienen a sus hijos inmunizados, participan en screenings de detección del cáncer cervical o exámenes de sangre para las pruebas de colesterol, controlan sus dietas, hacen ejercicio regularmente para protegerse contra enfermedades cardiovasculares u osteoporosis (traducido por los autores) $\mathbf{4 0 ( 6 9 )}^{\mathbf{4}}$.

Es preciso resaltar, como señalan los autores, que el tipo ideal de ciudadano 'sano' descrito difiere sustancialmente entre hombres y mujeres. Las mujeres son vistas más como un recurso para la reproducción (y, por ello, la importancia de los programas en salud reproductiva) y el mantenimiento de otros ciudadanos 'sanos', mientras que los hombres quedan apartados de la vigilancia médica, ya que no existe equivalente a la ginecología para el estudio y tratamiento de los cuerpos masculinos. Estos solo adquieren entidad pública en tanto fuerza laboral o cuando forman parte de un cuerpo de defensa. Los hombres, como ciudadanos, no tienen la responsabilidad de cuidar la salud de los demás, más bien lo contrario, ellos tienen el derecho a la protección por parte de otros. Sin embargo, esta realidad se ha modificado durante las dos últimas décadas debido a que se expandieron los servicios de salud para los hombres, siendo el screening para el cáncer de próstata el símbolo de la vigilancia de la salud masculina 4 .

Participar en un programa de cribado implica que las personas deben ser escaneadas periódicamente - en un examen de mamas, en la visita anual al ginecólogo o en un examen colorrectal-. Como vimos en la sección anterior, esa práctica se rutiniza y, al hacerlo, va configurando un tipo de paciente responsable no solo del cuidado de su salud individual, sino también porque va convirtiéndose en un individuo responsable socialmente, demostrando ser un 'buen ciudadano' que vela por su salud. Como advierte Howson ${ }^{60}$, las prácticas preventivas se vuelven una suerte de 'obligación moral'.

Este tipo de obligación, en la que se incluye la participación en un tamizaje, fue tema de análisis, también, en relación con el embodiment femenino, o sea, la obligación que las mujeres sienten en cuanto al cuidado y respeto por sus cuerpos $^{61}$. Pero, al mismo tiempo, en esta rutina existen incertidumbres manifiestas y latentes. Las primeras están asociadas con las experiencias y percepciones de las mujeres sobre el cribado e incluyen distintos grados de ambigüedad en relación con el propósito del tamizaje, el significado de un resultado anormal y el riesgo generado por la búsqueda de anormalidades cervicales. Por el contrario, las cuestiones de incertidumbre latente se asocian con las experiencias y percepciones de los profesionales encargados de ofrecer a sus pacientes los cribados ${ }^{62}$.

Asimismo, otra dimensión examinada por la literatura ha sido cómo los discursos médicos con relación a los cribados cervicouterinos han modificado la percepción de las mujeres en cuanto a lo 'normal', el sentido de la obligación asociada al examen Papanicolau y un nuevo tipo de feminidad ${ }^{63}$. Por otro lado, estudios han mostrado la contracara de este aspecto. Mckie ${ }^{64}$, por ejemplo, analizó los discursos de la clase trabajadora blanca masculina relatados por las mujeres y percibió que el cuello del útero era representado como sitio de vigilancia y control estatal, profesional y masculino, mientras que Daykin y Naidoo ${ }^{65}$ examinaron cómo este tipo de vigilancia sobre los cuerpos toma por sentado las desigualdades de género y adapta sus estrategias para mantener el status quo y, en definitiva, siguen reproduciendo las desigualdades existentes. Por su parte, Green y col.66 han examinado el rol que juegan las tecnologías de la salud en el manejo de los cuerpos de las mujeres de mediana edad y la manera en que estas las interpretan.

\section{Discusión}

En las dos últimas décadas del siglo XX, a diferencia del período anterior, los tamizajes 
quedaron reducidos a un puñado de programas adaptados a enfermedades, como algunos tipos de cáncer o a la vigilancia de la maternidad y la niñez, en los que se pone en juego el futuro. Antiguamente, el objetivo del cribado masivo era operar como estrategia para intentar demorar la aparición de la enfermedad o directamente detener su progresión, pero se modificó cuando los factores de riesgo comenzaron a utilizarse como predictores de enfermedades. A partir de entonces, ese nuevo raciocinio se extendió a otro tipo de enfermedades. Actualmente, el foco de atención para las enfermedades no transmisibles está centrado no tanto en la enfermedad en sí misma, sino en el estado semipatológico prepatogénico. Como el caso del cáncer, cada vez se confunden más las líneas divisorias entre lo patológico y el riesgo de enfermar, y la idea de riesgo refuerza, aún más, la delimitación de la vigilancia médica sobre ciertos grupos poblacionales en los que se ejerce un mayor control. Por ejemplo, se monitorea más a la población obesa porque pertenece a un grupo con mayor riesgo de enfermedades cardiovasculares en relación con la población general. Desde este paradigma, aún hegemónico de la biomedicina, las fronteras entre enfermos y sanos son cada vez más borrosas. Ahora todos, enfermos y sanos, estamos bajo la supervisión constante de la medicina. Más aún, los factores de riesgo existen en una relación móvil con otros riesgos, apareciendo y desapareciendo, cruzando espacios dentro y fuera del espacio corporal ${ }^{19}$.

La práctica clínica también se ha modificado a lo largo del tiempo. En la primera mitad del siglo XIX, centraba su mirada en las lesiones patológicas que se encontraban en el interior del cuerpo. En cambio, en la segunda mitad de ese siglo, estuvo marcada por la fisiopatología, que acercó el saber médico al de las ciencias 'duras' en el dominio de lo biológico. Por lo que, a partir de la segunda mitad del siglo XX, las enfermedades no transmisibles empiezan a ser definidas como consecuencia de factores de riesgos, más que como lesiones detectables en el organismo, y se definen, por primera vez en la historia, a partir de parámetros numéricos, como en el caso de la presión arterial. De esta manera, los estudios epidemiológicos comienzan a trazar las fronteras entre lo normal y lo patológico ${ }^{67}$. La enfermedad ya no está localizada en el cuerpo, sino en la población; sus causas son más externas que internas; multifactoriales más que unicausales, además, el locus de tratamiento ya no está situado en la cama del paciente, sino en la atención ambulatoria continua $^{68}$. Con la ayuda de los cribados poblacionales, entendidos como una estrategia de la salud pública y no solo como un examen clínico, estos cambios producidos en la práctica clínica se reforzaron. Ante ello, la realidad del cuidado de la clínica es compleja y, en ella, los tamizajes oportunistas se mezclan con el cuidado clínico cotidiano, ya sea por iniciativa del profesional de salud o por la del paciente. Tomando en consideración que el tamizaje y el diagnóstico o detección precoz de enfermedades son estrategias de salud diferentes, tienen, por tanto, implicaciones distintas. Mientras el tamizaje consiste en la observación de pacientes asintomáticos, la detección precoz refiere a cuando el paciente ya presenta signos y/o síntomas. Por ejemplo, luego de un estudio de viabilidad y costo-efectividad, Brasil decidió no implementar un tamizaje masivo y, mientras tanto, aplica la estrategia de diagnóstico precoz, apuntando a consolidar las bases necesarias para la implementación a futuro de un programa de tamizaje masivo ${ }^{53}$. Sin embargo, ambas estrategias generan un proceso de medicalización social que puede generar intervenciones diagnósticas y terapias excesivas y, a veces, dañinas ${ }^{69,70}$. Se puede citar el uso de nuevas tecnologías diagnósticas, como la ecografía mamaria, que nos permite mejorar sustancialmente la visión de esa parte del cuerpo. Hallazgos frecuentes con este tipo de técnicas son los nódulos sólidos. De acuerdo con las características ecográficas 
y el contexto clínico, el médico puede solicitar un seguimiento mediante estudios por imágenes o, después de la evaluación inicial, puede indicar una biopsia, cuyo resultado puede determinar que el nódulo estudiado corresponde a un fibroadenoma, que es el tumor benigno de mama más frecuente. En este caso, los nódulos no son una enfermedad en sí misma, pero son tratados como tal, y la mujer, en la mayoría de los casos, fue intervenida. No obstante, en el caso de la detección de un ganglio linfático intramamario, que es una condición que no produce síntomas ni es peligroso, el diagnóstico de este tipo de condiciones 'anormales' genera múltiples consecuencias para los pacientes como, por ejemplo, angustia e incertidumbre. En este contexto, se produce el reciente reconocimiento de la iatrogenia como una importante causa de mala salud ${ }^{71}$, lo que dio origen, entre los médicos generalistas europeos, al concepto y a la práctica de la prevención cuaternaria, relacionada con toda acción que atenúa o evita las consecuencias del intervencionismo médico excesivo $\mathbf{7 2}$.

La contracara de este proceso se presenta en las alteraciones en la percepción y las prácticas de cuidado que generan los tamizajes en los pacientes, produciendo diferentes situaciones, que fueron analizadas por la literatura. Esos 'buenos ciudadanos', que recurren a la consulta anual - clínica o ginecológica- pueden ser diagnosticados de cáncer entre una consulta y otra. Por consiguiente, esta situación relativamente frecuente causa una sensación de impotencia, dolor y perplejidad por el diagnóstico inesperado, especialmente por haber actuado como un paciente responsable. Pero también sucede lo contrario: la rutinización de la consulta clínica y los resultados normales en un tamizaje generan diferentes impactos en las personas. Uno de ellos es la ansiedad asociada a la espera de los resultados de un tamizaje ${ }^{73,74}$, así como recibir resultados 'sin riesgo'. La literatura ha puesto su foco de atención en la preocupación de que tales resultados puedan transmitir una sensación de 'falsa seguridad' $75 \mathrm{y}$, a su vez, desencadenar un efecto de 'certificado de salud'76.

Otra de las consecuencias de la rutinización de la consulta médica es que puede generar un exceso de demanda de pruebas diagnósticas por parte de la población debido al temor a la posibilidad de enfermar, a veces, sobrecargando los sistemas de salud con estudios innecesarios. Además, son frecuentes las quejas de las mujeres en relación con las visitas ginecológicas, es decir, cuando el médico indica un papanicolaou y no indica una colposcopia en la misma consulta. La información que proporcionan los profesionales de salud no siempre es clara y suficiente y los medios masivos de comunicación frecuentemente difunden mensajes confusos y contradictorios. Un estudio ha mostrado los problemas que han suscitado los screenings masivos que deberían tenerse en consideración a la hora de implementar esta estrategia de la salud pública: el enojo que sienten los pacientes decepcionados por estos programas; síntomas que son ignorados por la creencia de que los tamizajes ofrecen una protección completa; la responsabilidad que se le imputa al personal de la salud por problemas que, en realidad, son inherentes al cribado, y los malos entendidos que se producen sobre los beneficios, los límites y los costos de un tamizaje masivo77. Como ya se ha advertido, cuando se adoptan estrategias poblacionales, estas deben evitar la incomodidad y el dolor, además de ser mínimamente invasivas, socialmente aceptadas ${ }^{58} \mathrm{y}$ de bajo costo.

\section{Consideraciones finales}

Esta revisión buscó traer al debate académico una reseña de la literatura sobre una estrategia de la salud pública contemporánea denominada tamizajes masivos. También pretendió poner de manifiesto los aspectos sociales que emergieron a partir de la implementación de este tipo de estrategia, además, 
buscó exponer tres dimensiones que, consideramos, rompen con la lógica tradicional de comprensión de enfermedades, de la práctica clínica y del cuidado por parte de los pacientes y, al mismo tiempo, trató de integrar esas dimensiones en una narrativa común.

Como se ha mostrado, los tamizajes masivos han colaborado en invertir una concepción fuertemente arraigada en la forma tradicional de comprensión de las enfermedades, al poner su foco de atención más en la prevención primaria que en la secundaria, más en la detección de riesgos que en identificar signos tempranos de una enfermedad. En consecuencia, este desplazamiento generó una disminución en los umbrales para definir enfermedad y rompió con la distinción médica tradicional entre enfermos y sanos. Cuando la práctica del screening se convirtió en hegemónica en la sociedad, la práctica clínica se modificó al buscar enfermedades en personas asintomáticas y, al mismo tiempo, cambió los comportamientos de los pacientes, que pasaron a ser responsables del cuidado de sí mismos. En otras palabras, se produce una especie de 'transferencia de responsabilidad' de la medicina al individuo. Adicionalmente, el cuidado de sí mismo se transformó en un valor moral a partir del cual las personas comienzan a ser juzgadas.

\section{Contribuciones}

Los autores han contribuido, de igual manera, en la idea de elaborar una revisión de la temática, en el análisis de las obras y su interpretación. También han trabajado en el borrador y revisión crítica del contenido y han aprobado la versión final del manuscrito.

\section{Agradecimientos}

Los autores agradecen al Grupo de Estudios Sociales en Tecnociencia y Salud (BIOMEDSCI), del Instituto de Medicina Social - Uerj, por su contribución en la discusión de este trabajo, así como reconocen las invaluables contribuciones de Clarice Rios, Luciana Hadid y María Belén Herrero en las diferentes versiones que fue adquiriendo este artículo, y de Anabella Poggio, a través de su colaboración en la corrección de estilo y revisión gramatical del español. 


\section{Referencias}

1. Forman D, Sierra MS. Cancer in Central and South America: Introduction. Cancer Epidemiol. 2016 sep; 44(supl)S3-S10.

2. World Health Organization. Global action plan for the prevention and control of noncommunicable diseases 2013-2020. Geneva: WHO; 2013.

3. Starfield B, Hyde J, Gérvas J, et al. The concept of prevention: a good idea gone astray? J Epidemiol Community Health. 2008; 62(7):580-583.

4. Marcus PM, Prorok PC, Miller AB, et al. Conceptualizing overdiagnosis in Cancer Screening. J Natl Cancer Inst. 2015 fev; 107(4).

5. Faulkner A. Resisting the screening imperative: patienthood, populations and politics in prostate cancer detection technologies for the UK. Sociol Health Illn. 2012 feb; 34(2):221-233.

6. Hogarth S, Hopkins M, Rodriguez R. A molecular monopoly? HPV testing, the Pap smear and the molecularisation of cervical cancer screening in the USA. Sociol. Health Illn. 2012; 34(2):234-250.

7. Gøtzsche PC, Nielsen M. Screening for breast cancer with mammography. Cochrane Database Syst Rev. 2011 enero; (1):1-15.

8. Arrossi S. Cancer registration and information systems in Latin America. Lancet Oncol. 2015; 16(4):1400-1401.

9. Espinola N, Maceira D, Palacios, A. Costo-efectividad de las pruebas de tamizaje del cáncer colorrectal en la Argentina. Rev. Acta Gastroenterol Latinoamerican. 2016; 46(1):8-17.

10. Arrossi S, Thouyaret L, Paul L. Prevención del cáncer cervicouterino: recomendaciones para el tamizaje, seguimiento y tratamiento de mujeres en el marco de programas de tamizaje basados en el test de VPH: actualización 2015. Buenos Aires: Instituto Nacional de Cáncer; 2015.
11. Gualdrini UA, Iummato LE. Cáncer colorrectal en la Argentina: Organización, cobertura y calidad de las acciones de prevención y control. Informe final de diciembre de 2011: Diagnóstico de situación de la Argentina. Buenos Aires: Instituto Nacional de Cáncer; 2012.

12. Cronin P, Ryan F, Coughlan M. Undertaking a literature review: A step-by-step approach. Br J Nurs. 2008; 17(1):38-43.

13. Parahoo K. Nursing Research - principles, process and issues. 2. ed. London: Palgrave Houndsmill; 2006.

14. Cohen G. Memory. In: Roth I, editor. The Open University's Introduction to Psychology. New Delhi: Elsevier; 1990. p. 570-620.

15. Harold L, Miller JR. Sage Encyclopedia of Qualitative Research Methods. California: Sage Publications; 2016.

16. Bunton R, Nettleton S, Burrows R. The sociology of health promotion: Critical analyses of consumption, lifestyle and risk. London: Routledge; 1995.

17. Arouca S. O dilema preventivista: contribuição para a compreensão e crítica da medicina preventiva. São Paulo: Unesp; 2003.

18. Raffle A, Gray M. Screening: evidence and practice. Oxford: Oxford University; 2009.

19. Armstrong D. Screening: mapping medicine's temporal spaces. In: The Sociology of Medical Screening Critical Perspectives, New Directions. Singapure: Blackwell Publishing; 2012.

20. Kaufman P. A screening the body: the pap smear and the mammogram. In: Lock M, Young A, Cambrosio A. Living and working with the new medical technologies. Cambridge: Cambridge University Press; 2000. p. 165-183. 
21. Gardner KE. Early detection: women, cancer, and awareness campaigns in the twentieth-century united states. Carolina do Norte: University of North Carolina Press; 2006.

22. Löwy I. Cancer, women, and public health: the history of screening for cervical cancer. Hist Ciênc Saúde-Manguinhos. 2010 jul; 17(1):53-67.

23. Gibbon S. Breast Cancer Genes and the Gendering of Knowledge. England: Palgrave Macmillan; 2007.

24. Klawiter M. The Biopolitics of Breast Cancer. Minnesota: University of Minnesota Press; 2008.

25. Deshazer MK. Mammographies: the Cultural Discourses of Breast Cancer Narratives. Michigan: University of Michigan Press; 2013.

26. Foucault M. Vigilar y castigar. Madrid: Biblioteca Nueva; 2012.

27. Amstrong D. The rise of surveillance medicine. Sociol Health Illn. 1995; 17(3):393-404.

28. Beck U. Risk Society: Towards a New Modernity. London: Sage; 1992.

29. Bunton R, Petersen A. Genetic Governance: Health, Risk and Ethics in the Biotech Era. London; New York: Routledge; 2005

30. Conrad P, Schneide J. Deviance and medicalization: from badness to sicknes. Philadelphia: Temple University Press; 1992.

31. Conrad P. The Medicalization of Society on the Transformation of Human Conditions into Treatable Disorders. Baltimore: The Johns Hopkins University Press; 2007.

32. Clarke AE, Shim Jk, Mamo L, et al. Biomedicalization: Technoscientific Transformations of Health, Illness, and U.S. Biomedicine. Am Sociol Rev. 2003; 68(2):161-194

33. Zola IK. Medicine as an Institution of Social Con- trol. Sociol Rev. 1972 nov; 20(4):487-504.

34. Armstrong D. Political Anatomy of the Body. Cambridge: Cambridge University Press; 1983.

35. Armstrong D. Public Health Spaces and the Fabrication of Identity. Sociol. 1993; 27(3):393-410.

36. Leavell H, Clark EG. Medicina Preventiva. Rio de Janeiro: McGraw-Hill; 1976.

37. Doll R, Hill AB. La mortalidad en relación con el hábito de fumar: diez años de observaciones sobre médicos británicos. In: Buck C, Llopis A, Nájera E, et al. El Desafío de la Epidemiología. Washington: OPS; 1988.

38. Jackson P. The development of a scientific fact.: the case of passive smoking. In: Bunton R, Nettleton S, Burrows R, editores. The sociology of health promotion Critical analyses of consumption, lifestyle and risk. London: Routledge; 1995.

39. Lupton D. The Imperative of Health. London: Sage; 1995.

40. Petersen A, Lupton D. The New Public Health. London: Sage; 1996.

41. Armstrong N, Eborall H. The sociology of medical screening: past, present and future. In: The Sociology of Medical Screening Critical Perspectives, New Directions. New Jersey: Blackwell Publishing; 2012. p. 1-17.

42. Faulkner A. Resisting the screening imperative: patienthood, populations and politics in prostate cancer detection technologies for the UK. Sociol Health Illn. 2012; 34(2):221-233.

43. Hogarth S, Hopkins M, Rodriguez R. A molecular monopoly? HPV testing, the Pap smear and the molecularisation of cervical cancer screening in the USA. Sociol Health Ill. 2012; 34(2):234-250.

44. Hofmann B. On the value-ladenness of technology in medicine. Med Health Care Philos [internet]. 
2001 [acceso en 2018 jul 10]; 4(3):335-345. Disponible en: https://doi.org/10.1023/A:1012069919089.

45. Boenink M. Molecular medicine and concepts of disease: the ethical value of a conceptual analysis of emerging biomedical technologies. Med Health Care Philos [internet]. 2010 [acceso en 2018 jul 10]; 13(1):11-23. Disponible en: http://www.ncbi.nlm. nih.gov/pmc/articles/PMC2801843/.

46. Nordenfelt L. On Disability and Illness. A Reply to Edwards. Theoretical Med Bioethics. 1999; 20(2):181-189.

47. Nordenfelt L. Action, Ability and Health. Dordrecht: Springer Netherlands, 2000.

48. Nordenfelt L. On health, ability and activity: Comments on some basic notions in the ICF. Rev. Disability Rehabilitation. 2006; 28(23):1461-1465.

49. Lemoine M. Defining disease beyond conceptual analysis: an analysis of conceptual analysis in philosophy of medicine. Theoretical Med Bioethics. 2013; 34(4):309-325.

50. Rogers WA, Walker MJ. The Line-drawing Problem in Disease Definition. J Med Philos. 2017; 42(4):405-423.

51. Walker MJ, Rogers WA. Defining disease in the context of overdiagnosis. Med Health Care Philos. 2017; 20(2):269-280.

52. Doust J, Walker J, Rogers WA. Current Dilemmas in Defining the Boundaries of Disease. J Med Philosophy. 2017; 42(4):350-366.

53. Brasil. Ministério da Saúde. Secretaria de Atenção à Saúde. Rastreamento. Brasília, DF: MS; 2010.

54. Hofmann B. The overdiagnosis of what? On the relationship between the concepts of overdiagnosis, disease, and diagnosis. Med Health Care Philos. 2017; 20(4):453-464.

55. Gillespie C. The experience of risk as measured vul- nerability: health screening and lay uses of numerical risk. Sociol Health Illn. 2012; 34(2):194-207.

56. Donovan JL, Frankel SJ, Neal DE, et al. Screening for prostate cancer in the UK: Seems to be creeping in by the back door. BMJ. 2001; 323(7316):763-764.

57. Lehoux P, Denis JL, Rock M, et al. How medical specialists appraise three controversial health innovations: scientific, clinical and social arguments. Sociol Health Illn. 2010 enero; 32(1):123-139.

58. Rose G. The Strategy of Preventive Medicine. Oxford: Oxford Medical Publications; 1992.

59. Armstrong D. Chronic illness: a revisionist account. Sociol Health Illn. 2014; 36(1):15-27.

60. Howson A. Embodied obligation: the female body and health surveillance. In: Nettleton S, Watson J, editores. The Body in Everyday Life. London: Routledge; 1998.

61. Howson A. Cervical screening, compliance and moral obligation. Sociol Health Illn. 1999; 21(4):401425.

62. Howson A. Locating uncertainties in cervical screening. Health Risk Soc. 2001; 3(2):167-179.

63. Bush J. "It's just part of being a woman": cervical screening, the body and femininity. Soc Sci Med. 2000; 50(3):429-444.

64. Mckie L. The art of surveillance or reasonable prevention? The case of cervical screening. Sociol Health Illn. 1995; 17(4):441-457.

65. Daykin N, Naidoo J, Bunton R, et al. Feminist critiques of health promotion. In: The Sociology of Health Promotion. London: Routledge; 1995.

66. Green EE, Thompson D, Griffiths, F. Narratives of risk: Women at midlife, medical experts and health technologies. Health Risk Soc. 2002; 4(3):273-286.

67. Camargo KR. O paradigma clínico-epidemio- 
lógico ou biomédico. Rev Bras História Ciênc. 2013,6(2):183-195.

68. Armstrong D. Actors, patients and agency: a recent history. Sociol Health Illn. 2014; 36(2):163-174.

69. Tesser CD. Medicalização social (I): o excessivo sucesso do epistemicídio moderno na saúde. Interface. 2006; 10(19):61-76.

70. Tesser CD. Medicalização social (II): limites biomédicos e propostas para a clínica na atenção básica. Interface. 2006; 10(20):347-362.

71. Starfield B. Atenção primária: equilíbrio entre necessidades de saúde, serviços e tecnologia. Brasília, DF: Unesco; Ministério da Saúde; 2002.

72. Gervas J. Screening for serious illness: limits to the power of medicine. European J General Practice. 2002; 8(2):47-49.

73. Brett J, Bankhead C, Henderson B, et al. The psychological impact of mammographic screening: a systematic review. Psycho-oncology. 2005; 14:917938.
74. Orbell S, O' Sullivan I, Parker R, et al. Illness representations and coping following an abnormal colorectal cancer screening result. Social Science Med. 2008; 67:1465-1474

75. Pettigrew M, Sowden A, Sharp DL, et al. False-negative results in screening programmes: systematic review of impact and implications. Health Technol Assess. 2000; 4(5):1-33.

76. Tymstra T, Bieleman B. The psychosocial impact of mass screening for cardiovascular risk factors. Family Practice. 1987; 4(4):287-290.

77. Raffle AE. Information about screening: is it to achieve high uptake or to ensure informed choice. Health Expect. 2001 jun; 4(2):92-98.

Recibido en 08/01/2018

Aprobado en 04/07/2018

Conflicto de intereses: inexistente

Apoyo financiero: Consejo Nacional de Desarrollo Científico y

Tecnológico (CNPa) de Brasil, número de proyecto 401057/2015-1 